\title{
Targeting activated macrophages to identify the vulnerable atherosclerotic plaque
}

\author{
Lynne L. Johnson, MD ${ }^{a}$ \\ ${ }^{a}$ Columbia University, New York, NY \\ Received May 18, 2016; accepted May 18, 2016 \\ doi: 10.1007/s12350-016-0552-x
}

\section{See related article, pp. 862-871}

Hellberg and co-workers report the results of a preclinical study in mice designed to test the feasibility of targeting translocator protein (TSPO) with ${ }^{18} \mathrm{~F}$ FEMPA for the detection of atherosclerotic plaque inflammation. TSPO (formerly called peripheral benzodiazepine receptor) is a translocator protein that is ubiquitously expressed in mammalian tissue, localized to the outer mitochondrial membrane involved in cholesterol transport and expressed in activated macrophages. Because it targets activated macrophages, this radioligand is in theory a specific probe for inflammation imaging in atherosclerotic plaque. The investigators performed in vivo dynamic PET/CT in $3 \mathrm{LDLR}^{-/-} \mathrm{A}$ poB ${ }^{100 / 100}$ mice and 4 C57BL/6N (wild-type) mice to document whole-body biodistribution and kinetics. Additional mice (10 athero mice and 7 WT) were injected with radiotracer and sacrificed at 20 minutes and the thoracic aorta was removed for autoradiography and subsequently for immunohistology. Carotid endarterectomy tissue from 4 patients with recent cerebral ischemic events was also analyzed by in vitro autoradiography. While radioactive uptake as count density correlated with plaque macrophage density on ex vivo autoradiography for mouse tissue, ${ }^{18} \mathrm{~F}$-FEMPA was also taken up in normal arterial walls adjacent to the plaque and diffusely in the walls of large arteries of nonatherosclerotic WT mice, and consequently, there was no difference in radioactive uptake in plaque vs normal vessel. These findings were explained by high

Reprint requests: Lynne L. Johnson, MD, Columbia University, $630 \mathrm{~W}$ 168th St, PH 10-405, New York, NY 10032, USA; lj2129@ columbia.edu

J Nucl Cardiol 2017;24:872-5.

$1071-3581 / \$ 34.00$

Copyright (C) 2016 American Society of Nuclear Cardiology. constitutive expression of TSPO in endothelial cells and VSMCs in rodents. For the human autoradiographic and histological analysis, ${ }^{18}$ F-FEMPA uptake was also highest in macrophage-rich plaques with uptake also seen at lower levels in $\alpha$-SMA-positive areas but in vitro autoradiography was limited to the plaque region removed at endarterectomy, and therefore, adjacent nonatherosclerotic or remote vessel tissue was not available for study. While these preliminary studies do not stand as a positive proof of principle for the further development of this agent for in vivo PET imaging of inflamed atherosclerotic plaque in human subjects it is important to consider experimental and biological factors that may have affected the results and should be discussed before deciding on any possible future directions for this imaging ligand. The thoughtful study design and careful experimental approach carried out by Dr Hellberg and her co-investigators are instructive to considering the development or a targeted radiotracer to identify vulnerable atherosclerotic plaques.

In an era of limited resources, it is important to consider how an imaging test designed to identify atherosclerotic plaques with vulnerable characteristics prospectively might be applied in a cost-effective manner. ${ }^{1}$ Multimodality imaging combining either $\mathrm{CT}$ or MR for anatomical location and plaque tissue characterization with molecular targeting of disease activity using a PET-labeled ligand that binds a specific biological pathway known to destabilize plaque potentially provides the most complete non-invasive view into a patient's arteries, but this imaging approach is not inexpensive and therefore inappropriate to screen large numbers of patients at increased risk based on coronary risk factors. ${ }^{1}$ Nor is such a test technically simple, especially for the coronary arteries which require gating and careful image registration. The carotid arteries are easier targets because there is less motion and less tissue attenuation. The patient group for "screening" must be very carefully selected such as those with a first event to identify other lesions at risk for intervention. This approach is currently applied in the catheterization 
laboratory using IVIS to identify other at risk lesions remote from the culprit lesion. ${ }^{2}$

Recruitment of macrophages into the developing atheroma represents an important step in plaque development. In addition to inflammation, there are a number of other cellular pathways that have been identified as crucial in plaque evolution and have been targeted by radiotracers including adhesion molecules, cholesterol uptake, apoptosis, metalloproteinase and integrin expression during plaque growth, remodeling, and neoangiogenesis. ${ }^{3,4}$ Macrophage infiltration leading to a state of chronic inflammation in the vessel wall is recognized as a hallmark of plaque vulnerability both in the coronary and carotid circulation and therefore of high importance as an imaging target. The glucose analogue ${ }^{18} \mathrm{~F}-\mathrm{FDG}$ is taken up by metabolically active tissue using glucose as fuel which is the basis for its clinical utility in detecting metastatic disease. Inflamed tissue shares this high metabolic activity and inflammation is a hallmark of atherosclerotic plaque activity. Uptake of ${ }^{18} \mathrm{~F}-\mathrm{FDG}$ localized to carotid atherosclerotic plaque was first reported by Rudd et al in $2002 .^{5}$ Subsequent papers have explored the value of ${ }^{18} \mathrm{~F}$-FDG plaque imaging as a noninvasive biomarker to follow plaque inflammation over time and assess plaque stabilizing drug efficacy. ${ }^{6-9}$ Myocardial dependence on availability of exogenous glucose as a metabolic substrate makes visualizing the coronaries difficult to impossible with ${ }^{18}$ F-FDG despite dietary manipulations to switch myocardial substrate availability to fatty acids. In addition to the coronary circulation, other limitations to ${ }^{18} \mathrm{~F}-\mathrm{FDG}$ imaging in atherosclerosis include spillover from residual blood pool activity into the arterial walls, dependence of uptake on blood glucose levels that can be problematic in diabetics, and experimental data showing that hypoxia stimulates ${ }^{18} \mathrm{~F}$-FDG uptake in macrophages while proinflammatory cytokines stimulate uptake of ${ }^{18} \mathrm{~F}-\mathrm{FDG}$ in endothelial cells and smooth muscle cells. ${ }^{8,10}$ For these reasons, the search for alternative tracers targeting vascular inflammation is currently an area of active interest.

While the evolution of targeting TSPO for vascular inflammation imaging has an approximately seven year history, it has been known since 1984 that the translocator protein/peripheral benzodiazepine receptor (TSPO/ PBR) is highly expressed in macrophages, and in human tissue, the expression is $20 \times$ greater than in vascular smooth muscle cells. ${ }^{11,12}$ One of the earliest ligands developed for the TSPO was isoquinoline carboxamide PK11195 shown by ${ }^{3} \mathrm{H}$ autoradiography to be taken up in macrophage-rich regions of human carotid atherosclerotic vessel tissue preparations. ${ }^{11}$ Based on these reports, two subsequent pilot clinical imaging studies were performed in small groups of patients with arterial vasculitis and then in patients with carotid atherosclerosis using ${ }^{11} \mathrm{C}$ labeled PK11195 and imaging on a 16 -slice PET/CT scanner. ${ }^{13,14}$ In the first study, 6 patients with symptomatic large vessel vasculitis (giant cell arteritis, Takayasu's arteritis) were injected with ${ }^{11}$ C-PK11195 and PET imaging acquired over 60 minutes in list mode and re-binned followed by CT angiography. ${ }^{13}$ The CT scan matrix size was changed to match the PET scan and the images merged. Focal uptake in the arterial wall segments of the aortic arch was identified corresponding to areas of aortic wall thickening on CT angiogram in all 6 of the vasculitis patients and minor uptake in the region of the carotid bifurcation in one subject with atheroma on $\mathrm{CT}$ in that area. $^{13}$

Using the same radioligand in a second study, this group of investigators imaged the carotid arteries in patients with both symptomatic and asymptomatic carotid atherosclerotic diseases. ${ }^{14}$ In this study, 9 patients with recent cerebral vascular events and 27 asymptomatic patients with carotid stenosis $>50 \%$ were injected with ${ }^{11} \mathrm{C}$-PK111195 and using the same protocol underwent PET imaging followed by carotid CT angiography. From the registered PET/CT images, tracer uptake in plaques identified on CTA was quantified as SUV and plaque tissue densities measured as HUs. The investigators identified focal tracer uptake in plaques on the ipsilateral side associated with CNS event, and the mean T/B of $1.06 \pm 0.20$ was significantly higher than in the asymptomatic patients, $(0.86 \pm 0.11, \mathrm{P}=0.001)$; however, there was considerable overlap between values for the two groups (Figure 2 in reference ${ }^{14}$ ). The ex vivo autoradiography performed by incubating tissue acquired at carotid endarterectomy with ${ }^{3} \mathrm{H}-\mathrm{PK} 11195$ showed good co-localization of uptake with cells staining positive for TSPO and CD68. While the PET data alone showed uptake in carotid plaques of symptomatic patients, the signal was not very high compared to background. To obtain optimal diagnostic accuracy, parameters from both the PET and CT scans were combined. The authors point out limitations of the short half-life of C-11 requiring on-site cyclotron and high dosimetry from combined PET and CTA radiation exposure.

Based on these pilot PET studies, targeting activated macrophages in arterial vessel walls and the limitations of ${ }^{11} \mathrm{C}$ isotope, ${ }^{18} \mathrm{~F}$-FEMPA a novel secondgeneration TSPO radioligand was developed and applied to the task of identifying neuroinflammation in early Alzeheimer's disease. In this disease, TSPO is expressed on microglia and reactive astrocytes in addition to macrophages and activated microglia and macrophages are characteristic of early disease. Using tracer kinetic modeling, they identified differences among subjects in binding affinities and significant differences between the 
disease and non-disease groups were seen only in the high-affinity binding subjects. ${ }^{15}$

A summary of potentially favorable and unfavorable findings gleaned from these studies targeting TSPO with PET-labeled radioligands might include in the favorable column the following: targeting activated macrophages as one of the most important hallmarks of the vulnerable atherosclerotic plaque, a mitochondrial translocator protein abundantly found in activated macrophages, the findings from pilot human trials that PET-labeled radioligands targeting TSPO show focal uptake in arteritis, carotid atherosclerosis, and Alzheimer's disease confirmed by autoradiography. The unfavorable column includes the following: relatively low target uptake in atherosclerotic plaques imaged and need to augment diagnostic accuracy by combining CTA and PET parameters, and possible differences in uptake among patients due to differences in binding affinities.

Added to the unfavorable list are several of the findings reported in the current paper by Hellberg et al. These include her findings of diffuse uptake of ${ }^{18} \mathrm{~F}$ FEMPA in unaffected arterial walls adjacent to the plaque in the atherosclerotic mice as well as diffusely in the walls of large arteries of non-atherosclerotic WT mice and relatively slow clearance of lung activity. These findings may to some degree be due to the size of the mouse subject and biology unique to rodents. High constitutive expression of TSPO in endothelial cells and VSMCs and in brown adipose tissue surrounding the vessel in rodents has been reported. ${ }^{16}$ In human atherosclerotic tissue, TSPO was observed to be $20 \times$ greater than in vascular smooth muscle cells. ${ }^{12}$ The author comments that inability to differentiate plaque from normal aorta may also be due to "spill-over" from residual blood activity into the arterial walls which is also related to limited spatial resolution for PET imaging in rodents. While the physics of positron annihilation and design of PET camera favors a higher resolution than SPECT in human subjects, it actually favors a lower resolution in mice. ${ }^{17}$ PET radiotracers emit positrons from the nucleus which travel a calculated range from $\sim 0.5$ to $2 \mathrm{~mm}$ in tissue before annihilations (distance depending on the radionuclide). While the "blurring" effect of this short passage is not consequential in human imaging, it reduces the spatial resolution of micro-PET making it more difficult to detect very small targets such as atherosclerotic plaque in the aortic wall. It should be noted that in both of the human studies of ${ }^{11} \mathrm{C}$-PK11195 in large vessel arteritis and in carotid atherosclerosis, uptake of tracer in non-affected arterial wall was not commented on and the images shown do not suggest diffuse high vascular uptake of tracer was present. The additional finding in mice reported by Hellberg et al is the initial high lung uptake of ${ }^{18}$ F-FEMPA after injection which drops relatively slowly and by $30-40$ minutes remains higher than aorta in both the WT and atherosclerotic mice. The biodistribution of any radiotracer must be considered when selecting a probe with optimal characteristics for a specific target. If this target is in the thorax, then lung uptake is of considerable importance. In one clinical study reporting ${ }^{18}$ F-FEMPA in human subjects, the authors report only the brain imaging and make the statement that this radioligand "has displayed suitable pharmacokinetic properties in preclinical studies" but do not reference this comment so we do not know the lung uptake found in the presumed normal subjects comprising the preclinical study group. ${ }^{15}$ Species differences in distribution of peripheral-type benzodiazepine binding sites in lungs were reported. ${ }^{18}$

In concluding, the question arises as to whether further development of ${ }^{18} \mathrm{~F}$-FEMPA to identify vulnerable atherosclerotic plaque in patients is warranted. Replacing ${ }^{11} \mathrm{C}$ with ${ }^{18} \mathrm{~F}$ PET radiolabel overcomes the limitation of ultra-short half-life and need for on-site cyclotron. It is not known, however, whether the change in structure of the protein will change the plaque uptake of the radioligand reported in the paper by Gaemperli et al and needs to be addressed. Regardless of the radioisotope and radioligand, full characterization of atherosclerotic plaque on a PET scan requires combining anatomical location and plaque tissue characteristics with radiotracer uptake. ${ }^{19}$ Dosimetry from CT scans has been reduced with improved acquisition parameters and CT design over the 16-slice scanner used in the 2011 paper. Remaining unknowns include possibly unfavorable biodistribution ${ }^{18}$ F-FEMPA namely lung uptake which would make imaging the aorta problematic and possible variability among patients in binding affinities of ${ }^{18}$ F-FEMPA for macrophages in atherosclerotic plaque as was seen in brain imaging paper. And lastly, comparison of TSPOtargeted PET tracers with newly reported results from other atheroma-targeted PET radioligands is ultimately the most important indication of how this radioligand would fare in finding a niche for localizing at risk lesions in selected patients with very high risk for future events.

\section{Disclosure}

The author has no conflict of interest to report.

\section{References}

1. Braunwald E. Noninvasive detection of vulnerable coronary plaques. J Am Coll Cardiol 2009;54:49-57.

2. Mintz GS. Clinical utility of intravascular imaging and physiology in coronary artery disease. J Am Coll Cardiol 2014;64:207-22.

3. Kusters DH, Tegtmeir J, Schurgers LJ, Reutelingsperger CP. Molecular imaging to identity the vulnerable plaque-from basic research to clinical practice. Mol Imaging Biol 2012;14:523-33. 
4. Narula J, Nakano M, Virmani R, Kilodgie FD, Petersen R, Newcomb R, et al. Histopathologic characteristics of atherosclerotic coronary disease and implications of the findings for the invasive and noninvasive detection of vulnerable plaques. J Am Coll Cardiol 2012;61:1041-51. doi:10.1016/j.jacc.2012.10.054.

5. Rudd JH, Warburton EA, Fryer TD, Jones HA, Clark JC, Antoun $\mathrm{N}$, et al. Imaging atherosclerotic plaque inflammation with [18F]fluorodeoxyglucose positron emission tomography. Circulation 2002;105:2708-11.

6. Tawakol A, Migrino RQ, Bashian GG, Bedri S, Vermylen D, Cury $\mathrm{RC}$, et al. In vivo ${ }^{18} \mathrm{~F}$-fluorodeoxyglucose positron emission tomography imaging provides a noninvasive measure of carotid plaque inflammation in patients. J Am Coll Cardiol 2006;48:181824.

7. Silvera SS, Aidi HE, Rudd JH, Mani V, Yang L, Farkouh M, et al. Multimodality imaging of atherosclerotic plaque activity and composition using FDG-PET/CT and MRI in carotid and femoral arteries. Atherosclerosis 2009;207:139-43.

8. Bucerius J, Mani V, Moncrieff C, Machac J, Fuster V, Farkouh ME, et al. Optimizing ${ }^{18}$ F-FDG PET/CT imaging of vessel wall inflammation: The impact of ${ }^{18} \mathrm{~F}$-FDG circulation time, injected dose, uptake parameters, and fasting blood glucose levels. Eur J Nucl Med Mol Imaging 2014;41:369-83.

9. Rudd JHF, Narula J, Strauss W, Virmani R, Machac J, Klimas M, et al. Imaging atherosclerotic plaque inflammation by fluorodeoxyglucose with positron emission tomography. J Am Coll Cardiol 2009;55:2527-35. doi:10.1016/j.jacc.2009.12.061.

10. Folco EJ, Sheikine Y, Rocha VZ, Christen T, Shvartz E, Sukhova $\mathrm{G}$, et al. Hypoxia but not inflammation augments glucose uptake in human macrophages. J Am Coll Cardiol 2011;58:603-14. doi: 10.1016/j.jacc.2011.03.044.

11. Zavala F, Haumont J, Lenfant M. Interaction of benzodiazepines with mouse macrophages. Eur J Pharmacol 1984;106:561-6.
12. Bird JLE, Izquierdo-Garcia D, Davies JR, Rudd JHF, Probst KC, Figg N, et al. Evaluation of translocator protein quantification as a toll for characterizing macrophage burden in human carotid atherosclerosis. Atherosclerosis 2010;210:388-91.

13. Pugliese F, Gaemperli O, Kinderlerer AR, Lamare F, Shalhoub J, Davies AH, et al. Imaging of vascular inflammation with [11C]PK11195 and positron emission tomography/computed tomography angiography. J Am Coll Cardiol 2010;56:653-61. doi: 10.1016/j.jacc.2010.02.063.

14. Gaemperli O, Shalhoub J, Owen DR, Lamare F, Johansoon S, Fouladi $\mathrm{N}$, et al. Imaging intraplaque inflammation in carotid atherosclerosis with 11C-PK1195 positron emission tomography/computed tomography. Eur Heart J 2012;33:1902-10.

15. Varrone A, Oikonen V, Forsbert A, Joutsa J, Takano A, Solin O, et al. Positron emission tomography imaging of the $18-\mathrm{kDa}$ translocator protein (TSPO) with [18F] FEMPA in Alzheimer's patients and control subjects. Eur $\mathrm{J}$ Med Mol Imaging 2015;42:438-46.

16. French JF, Matlib MA. Identification of a high-affinity peripheraltype benzodiazepine binding site in rat aortic smooth muscle membranes. J Pharmacol Exp Ther 1988;247:23-8.

17. Ganguly BN, Mondal NN, Nandy M, Roesch F. Some physical aspects of positron annihilation tomography: A critical review. J Radioanal Nucl Chem 2009;279:685-98.

18. Mak JCW, Barnes PJ. Peripheral type benzodiazepine receptors in human and guinea pig lung: Characterization and autoradiographic mapping. J Pharmacol Exp Ther 1989;252:880-5.

19. Motoyama S, Sarai M, Harigaya H, Anno H, Inoue K, Hara T, et al. Computed tomography angiography characteristics of atherosclerotic plaques subsequently resulting in acute coronary syndrome. $\mathrm{J}$ Am Coll Cardiol 2009;54:49-57. doi:10.1016/j.jacc.2009.02.068. 